\title{
System Analysis and Design of mmW Mobile Backhaul Transceiver at $28 \mathrm{GHz}$
}

\author{
Giuseppe Destino*, Olli Kursu*, Saila Tammelin ${ }^{\dagger}$, Jari Haukipuro*, \\ Marko Sonkki*, Timo Rahkonen*, Aarno Pärssinen*, Matti Latva-aho*, Aki Korvala ${ }^{\dagger}$, Marko Pettissalo ${ }^{\dagger}$ \\ *University of Oulu, Oulu, Finland \\ ${ }^{\dagger}$ Nokia, Oulu, Finland \\ email: \{giuseppe.destino, olli.kursu, jari.haukipuro, marko.sonkki, timo.rahkonen, \\ aarno.parssinen, matti.latva-aho\}@oulu.fi, \{aki.korvala, marko.pettissalo, saila.tammelin\}@nokia.com
}

\begin{abstract}
In the next generation of mobile network, $5 \mathrm{G}$, mmwave $(\mathrm{mmW})$ communication is considered one of the main disruptive technologies to increase data rates and improve spectrum efficiency. Wireless backhaul with stationary or moving nodes is one of the best candidate use-cases. This paper provides a comprehensive analysis on the architecture and design of mmW transceiver with automatic gain control (AGC) for mobility management. The focus is on the $\mathrm{RF}$ component requirements, especially, power amplifiers, low-noise amplifier and antennas as well as on their impact on the link-budget. Results are provided based on real figures of commercial components.
\end{abstract}

\section{INTRODUCTION}

One of the technology drivers of the next generation of mobile networks (so-called 5G) is the communication at the high carrier frequencies, i.e., millimeter wave $(\mathrm{mmW})$ frequency band, also E-band [1]. In the E-band the available spectrum is higher than in lower bands, quasi-optical radio propagation properties [2] allowing very directional communications and, finally, lower spectrum license costs leading to a lower total cost of ownership and lower cost per bit. The World Radiocommunication Conference (WRC)-15 decided frequency bands in the E-band for the future development of International Mobile Telecommunications (IMT) services [3], and more specifically, mobile bands are located between 24.25 and $86 \mathrm{GHz}$. The spectrum between 26.5 and $29.5 \mathrm{GHz}$ is the band proposed for the Winter Olympics in Korea, of which $27.5-28.5 \mathrm{GHz}$ is used for demonstrations [4].

In this paper we target the specific design of a $\mathrm{mmW}$ radiounit at $28 \mathrm{GHz}$, with a special emphasis on the radio frequency (RF)-Front-end (FE) capable of beamforming. Unlike the $60 \mathrm{GHz}$ technology, e.g, WiGig, chipset availability for $28 \mathrm{GHz}$ $\mathrm{RF}$ is limited. Samsung led developments in the $28 \mathrm{GHz}$ band together with NYU and developed early prototypes. After the WRC-15, many chipset vendors started to develop $28 \mathrm{GHz}$ technologies, which led to the announcement of Intel and Qualcomm plans to support $28 \mathrm{GHz}$ in their $5 \mathrm{G}$ chipsets in 2018. NEC released a massive MIMO antenna at $28 \mathrm{GHz}$ band with 500 elements, which will be used in the earliest deployment of $5 \mathrm{G}$ in Japan.
Table I

BASE-BAND PERFORMANCE

\begin{tabular}{l|cc|cc|cc|cc} 
Modulation & \multicolumn{2}{|c|}{ QPSK } & \multicolumn{2}{c|}{ 16QAM } & \multicolumn{2}{c|}{ 64QAM } & \multicolumn{2}{c|}{ 256QAM } \\
\hline Coding rate & \multicolumn{2}{|c|}{0.85} & \multicolumn{2}{|c|}{0.85} & \multicolumn{2}{c|}{0.85} & \multicolumn{2}{c}{0.85} \\
\hline Rank & 4 & 8 & 4 & 8 & 4 & 8 & 4 & 8 \\
\hline Rate [Gbps] & 0.6 & 1.2 & 1.2 & 2.4 & 1.8 & 3.6 & 2.4 & 4.9
\end{tabular}

Recently, a 28GHz IC was published by IBM and Ericsson representing state-of-the-art in the integrated solutions for $5 \mathrm{G}$. Our design is one of the first prototype for mobile-backhaul connecting, for instance, bus or trains via high directional communication link [4], [5]. In addition to RF energy efficiency and high data rate requirements, the proposed design includes features for mobility management at the hardware level, namely, the Automated Gain Control (AGC).

As the design is focused at the $28 \mathrm{GHz}$, a carrier frequency new for mobile applications, new challenges are met, especially in the selection of components and architecture in order to minimizes energy losses. Special care is, indeed, required for the selection of the RF-beamformer architecture and $\mathrm{mmW}$ Power-amplifier (PA).

The paper is organized as follows. In Section II an overview of the transceiver architecture, including base band (BB) and RF-FE is provided. Section III is focused on the analysis of the component requirements and of the transmitter and receiver paths. Also, it includes the description of a transceiver implementation with state-of-the-art commercial components. Section III tackles the specific feature of AGC for mobility and Section IV shows the resulting link budget analysis including realistic component loss figures. Finally, in Section $\mathrm{V}$ conclusions and remarks are drawn.

\section{MMW BACKHAUL RADIO UNIT}

A $\mathrm{mmW}$ radio unit for mobile backhaul application at $28 \mathrm{GHz}$ is considered. Key requirements are long-coverage (a few hundreds of meters), $>2$ Gbps data-rate, beamforming and mobility management. Starting from the data-rate requirements, thus the $\mathrm{BB}$ capacity, we rely on multipleinput-multiple-output (MIMO)-orthogonal frequency-division multiplexing (OFDM) technology with hybrid digital-radio frequency beamforming capabilities. More specifically, the MIMO-OFDM solution follows a classical implementation of 
a Long Term Evolution (LTE) modem including: $i$ ) scrambling, ii) quadrature modulation, iii) layer mapping, iv) MIMO precoding, $v$ ) frequency resource mapping and $v i$ ) OFDM signal generation. Relying on the pre-standard 5GTF specifications [4], the intercarrier spacing of the OFDM signal is $\Delta_{f}=75 \mathrm{kHz}$ and the number of used subcarriers is 1200 . Therefore, using this numerology as baseline, the transmission bandwidth (BW) is $100 \mathrm{MHz}$. Table I shows the maximum data-rate capacity of the proposed $\mathrm{BB}$ solutions using different MIMO and modulation configurations.

It can be noticed that data-rate requirements are met already with 16QAM and 8 MIMO layers. Alternatively, it is necessary high order modulation, e.g. 256QAM with 4 spatial layers.

The design principle of the mmW RF unit as well as the PCB layout is depicted in Figure 1. More specifically, the transceiver is designed for a time-division-duplex (TDD) communication where the transmission and receiving chain are not simultaneously utilized but separated by a RX/TX switch. Also, it can be noted that the RF beamformer is implemented with phase-shifters and without the amplitude control. This allows less losses and calibration control.

The phase shifters are controlled by a RF beamformer logic and are used to modify the signal phase at the input (or) output of the each antenna element. Another important aspect of this design is the distribution line connecting the input/output signal to $N_{A}$ antennas. In order to minimize the losses, we selected an architecture with a cascade of $L$ stages of power-split, where $L=\log _{2}\left(N_{A}\right)$. A dual-stage power amplification, namely, one at the beginning and the other at the end of the stage $L$, yields a good trade-off between cost, power compensation and energy consumption. Finally, a mixer is used to lift-up (or move down) an intermediate frequency (IF) used by the Digital Front-End (DFE) to the final $\mathrm{mmW}$ carrier.

\section{DESIGN OF THE TX AND RX PATHS}

In this section we focus on the description and analysis of the RF components for an mmW transceiver operating in the range of $26-30 \mathrm{GHz}$ in a Time-Division-Duplex (TDD) mode. We shall not refer to specific commercial products, however, we highlight key requirements and characteristics.

To begin with, let us consider the TDD system requirement, which directly implies that antennas as well as all reciprocal components of the signal paths are shared between the TX and RX chains. Also, that enough isolation (e.g. $40 \mathrm{~dB}$ ) exists between the TX and RX paths, which is achieved with AlGaAs PIN diode switches.

Next, we consider the frequency range, the Ka-band. In this regard, only a sparse selection of commercial components is available, today. Special care is needed for the choice of the PA and Low noise amplifier (LNA). For the former our main criteria is to achieve high output power and gain. For the latter gain and noise figure were the key discriminating factors.

\section{A. Transmitter path}

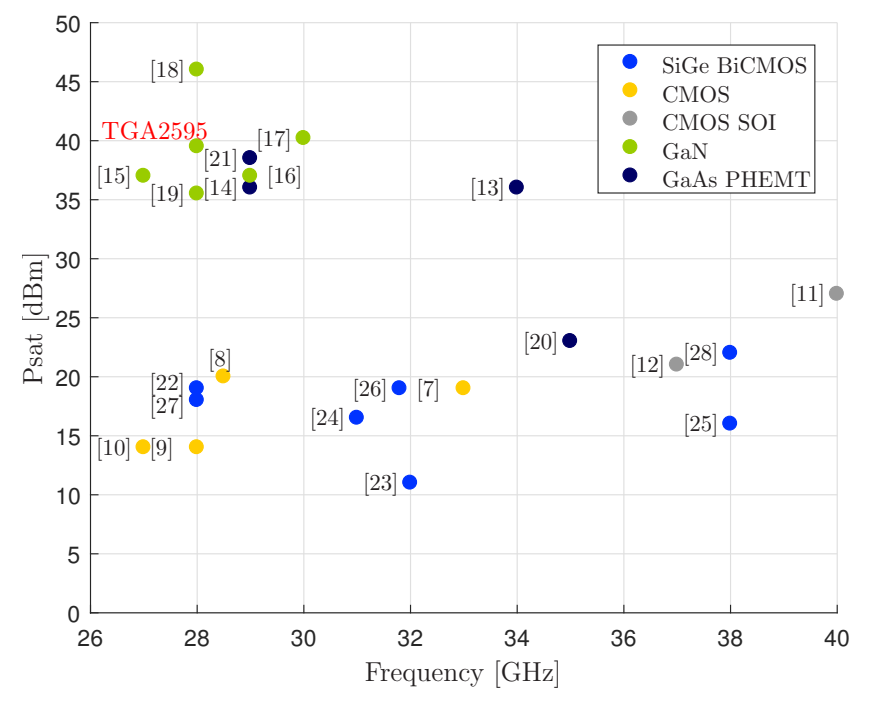

Figure 2. Classification of the power amplifier operating in the $26-40 \mathrm{GHz}$ spectrum.

On transmit path the signal is upconverted from $1-6 \mathrm{GHz}$ IF frequency to the final TX frequency of $26-30 \mathrm{GHz}$ using a commercial subharmonically pumped $21-31 \mathrm{GHz} \mathrm{GaAs}$ mixer with an integrated LO amplifier.

Subharmonic pumping alleviates the requirement for the $\mathrm{LO}$ generation since the final RF frequency is $2 \mathrm{xLO}+\mathrm{IF}$, which means that the synthesizer can operate at less than half of the final operating frequency. A three stage Wilkinson power divider network is used to divide the signal to 8 antenna modules. The signal is amplified before the power divider network using two GaN power amplifiers. Each antenna branch contains digitally controlled passive phase-shifter with finite phase resolution.

On the selected frequency band, GaN (Gallium nitride) on SiC (Silicon carbide) technology offers significantly higher output power levels compared to other technologies. Fig. 2 shows a comparison of different power amplifier technologies. Also, it can be seen that only GaN and GaAs technologies can provide appropriate output power levels for our application.

Thus, we propose the utilization of GaN amplifiers, e.g. TGA2595. This specific amplifier can, in fact, provide $30 \mathrm{~dB}$ gain and output third order intercept point (OIP3) of $48.5 \mathrm{~dB}$ and power-added efficiency (PAE) of $24 \%$. However, when using higher order QAM schemes, $10 \mathrm{~dB}$ or more backoff is required to achieve the required error vector magnitudes (EVM), reducing the PAE significantly.

\section{B. Receiver path}

In the receiver path, one of the most important components is the LNA. Likewise the PA in the TX path, the choice of components is limited due to the operation frequency. In the proposed design, we selected GaAs MMIC operating in the band $22-38 \mathrm{GHz}$. While GaAs and GaN amplifiers have similar noise performance, GaAs amplifiers have significantly 

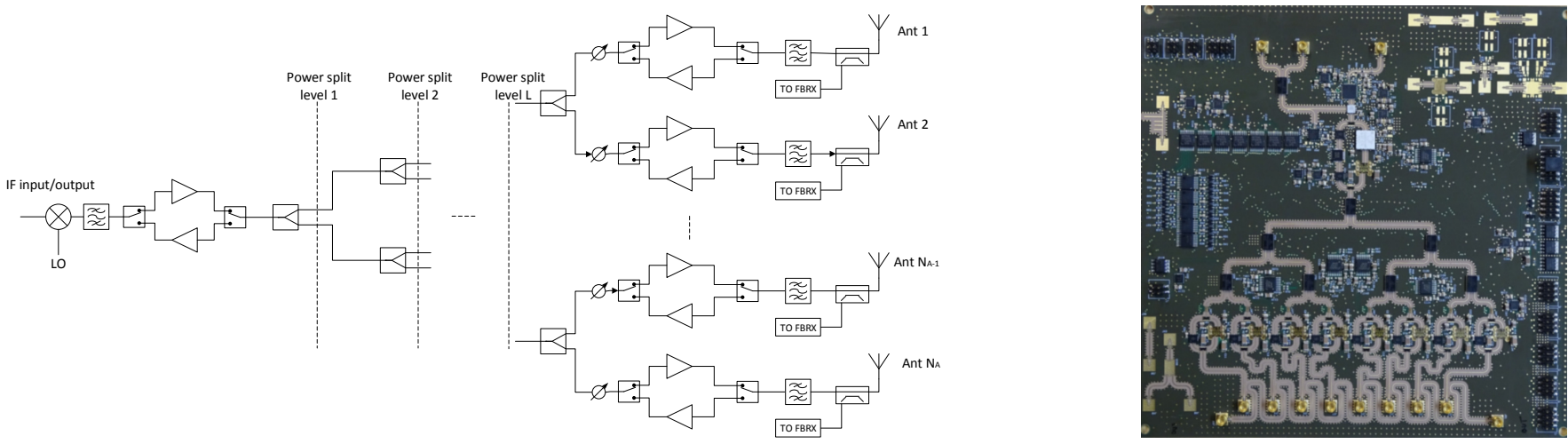

Figure 1. Implementation design of the $\mathrm{mmW}$ beamformer unit

lower cost. Additionally, the GaAs LNA requires only a single supply voltage. The LNA has a noise figure of $2.5 \mathrm{~dB}$ and a gain of $19 \mathrm{~dB}$. Receiver gain is controlled with a digital attenuator with 5-bit resolution located between the two LNAs following the power combining network. The receiver path also contains a commercial demodulating logarithmic amplifier that acts as a received signal strength indicator (RSSI) for the combined output. Passive reciprocal phase shifting, power division/combining and mixing are shared with the TX path.

\section{Power analysis of the TX and RX chains}

Figure 4 shows the cascaded gain and OIP 3 of the TX path as well as the cascaded gain and noise figure of the RX path with minimum and maximum attenuation values. Data is calculated from the component data sheets. Microstrip losses are simulated at $28 \mathrm{GHz}$ using a 2.5D EM simulator.

In the TX direction the power dividers cause power reduction, while in the RX direction the combiners sum up coherent $\mathrm{RX}$ signals, leaving just the losses of the transmission lines.

\section{D. $A G C$}

In order to allow mobility of the backhaul link, the receiver needs more input signal dynamic area than a fixed link. The signal has to be kept in a certain level at the ADC input of the receiver and, in particular, has to fit within the linearity region of the components in the RF chain. One method to enhance the dynamic range of the receiver is the utilization of AGC.

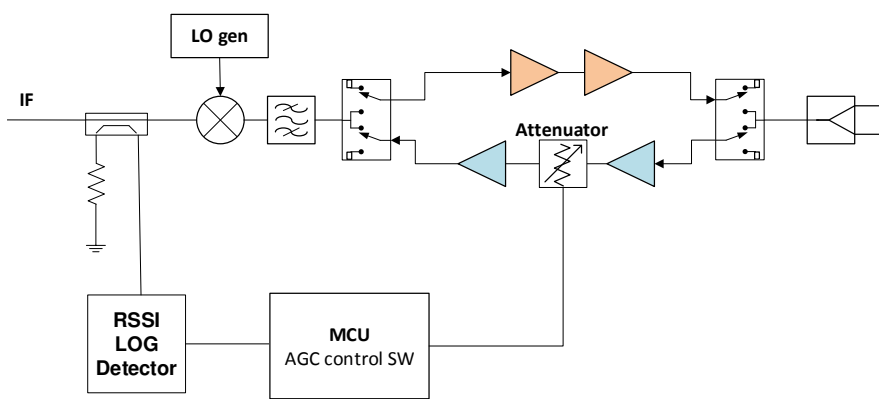

Figure 3. Block diagram of the AGC control loop including a digitally controlled attenuator and an RSSI circuit.
AGC can be implemented in several ways. Commonly, a solution is to consider the gain of the RF chain as a variable and controllable by using gain controlled amplifiers or attenuators. In our design, the AGC functionality is implemented after the power combining network, between two LNAs.

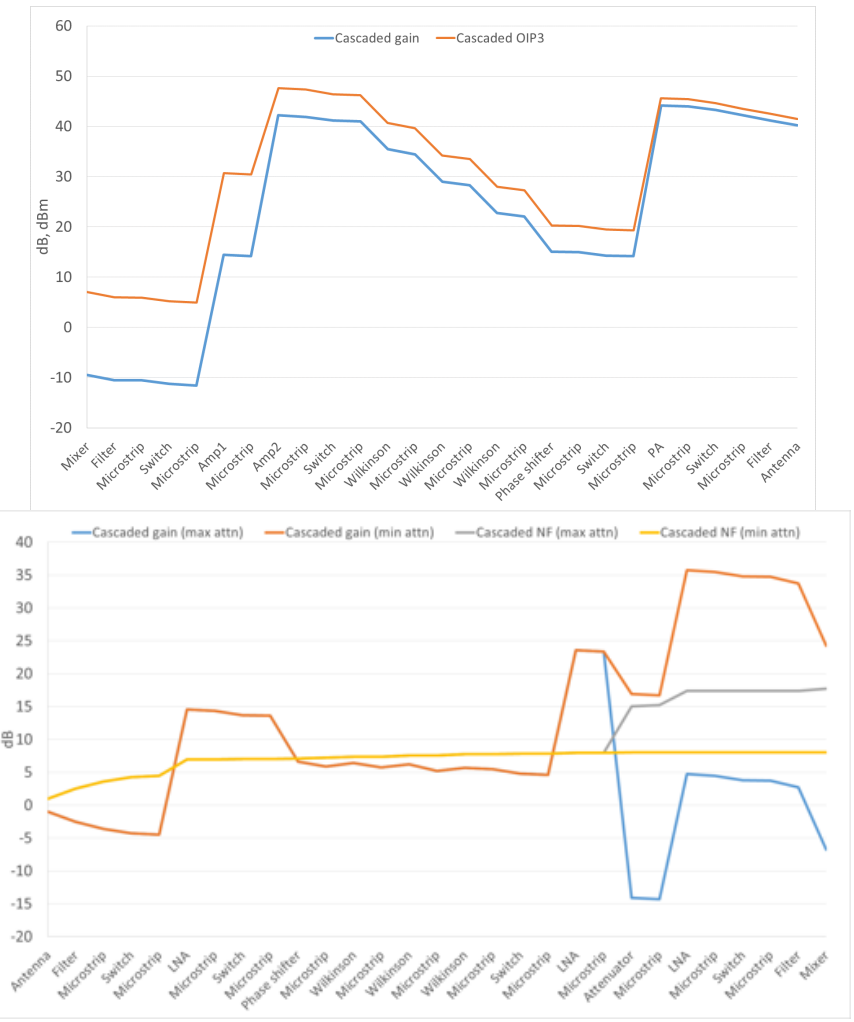

Figure 4. Cascade power analysis: (left) transmitter chain, (right) receiver chain.

The schematic is illustrated in Figure 3. It can be noticed that the IF signal is sampled by means of a microstrip directional coupler and each sample is fed to a RSSI log detector circuit. The gain control (attenuation) is carried out with an adjustable 5-bit attenuator in the RF part of the RX chain. The controlling routine is run by an external microcontroller unit (MCU). With this solution, the dynamic range of the receiver is estimated to be $31 \mathrm{~dB}$ with $64 \mathrm{QAM}$. 


\section{E. mmW Antenna Array}

In Figure 5 it is shown a linearly polarized $2 \times 2$ sub-array antenna structure with the coordinate system.
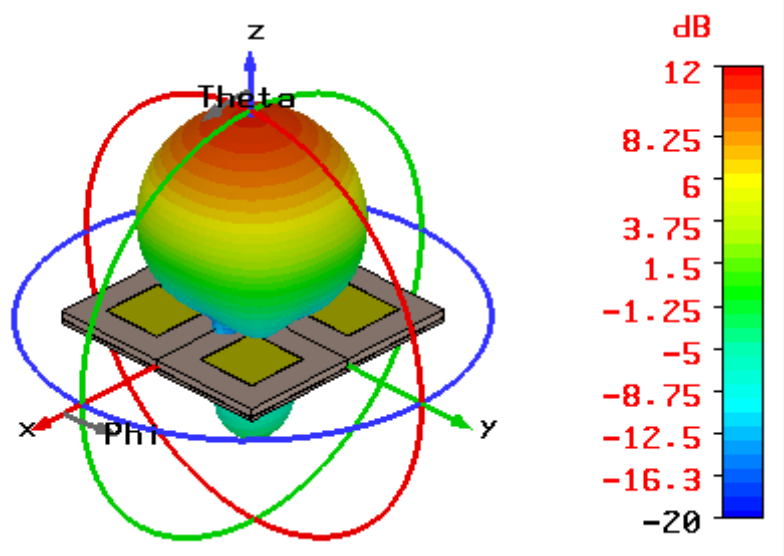

Figure 5. 2x2 element sub-array used as a basic radiating element in a planar antenna array at $28 \mathrm{GHz}$ center frequency.

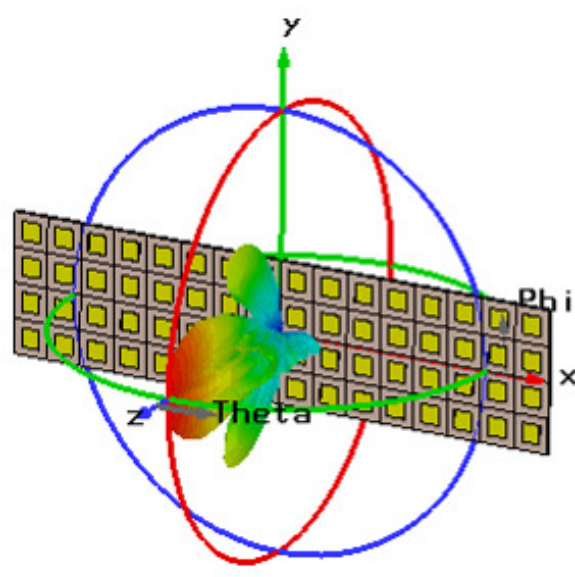

$\mathrm{dB}$
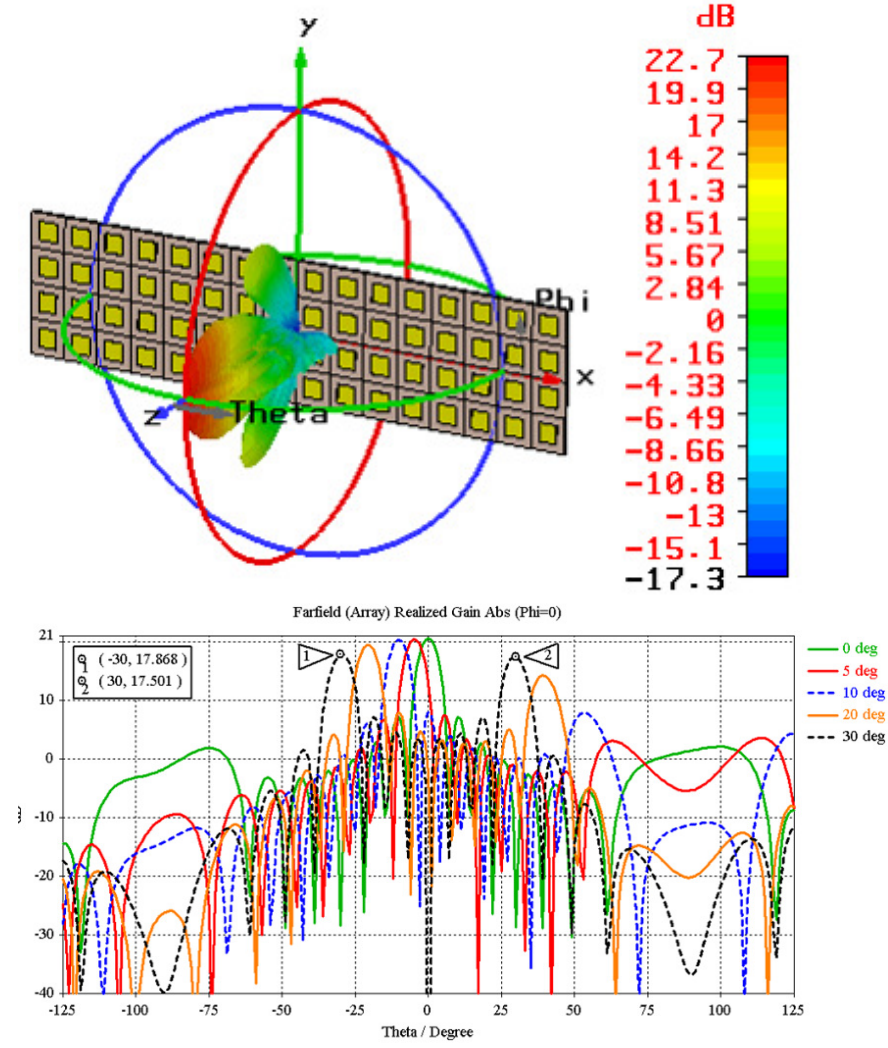

Figure 6. (Top)3D Main beam. (Down) Beamsteering in the azimuth domain.

The sub-array is designed for $28 \mathrm{GHz}$ center frequency with $10 \%$ relative $-14 \mathrm{~dB}$ impedance bandwidth. The maximum gain in broadside is $10.8 \mathrm{~dB}$.

The physical dimension of the sub-array is $(10.7 \times 10.7 \times$ 1.6) $\mathrm{mm}^{3}(\mathrm{~L} \times \mathrm{W} \times \mathrm{H})$, which is chosen based on the area of commercial power amplifiers available on the markets.
The $2 \times 2$ subarray is then utilized as the basic module for a planar antenna array. The phase-shifters of the RF beamformer are, in fact, connected to the radiating elements (patch antennas) in the sub-array. In total, the array includes 16 sub-arrays in form of $2 \times 8$ element modules. It results that the patch element spacing is still half a wavelength although the sub-array spacing is one lambda. The total form-factor of the planar antenna array is $9.6 \times 2.4 \mathrm{~cm}$.

In Figure 6 the beampattern of the array is illustrated. The azimuth resolution has been emphasized more than elevation, in fact, beams are narrower when $\phi=0$. Also, it can be noticed that by using sub-arrays in an array leads to increased aperture size compared to an antenna array where radiating elements are placed every half a lambda. However, grating lobes are likely to appear.

Throughout simulations, it is observed that $22.06 \mathrm{dBi}$ is the maximum array gain achieved in the broadside direction. Furthermore, due to the radiation capabilities of the $2 \times 2$ subarray, the scanning area in the azimuth and elevation domain is limited to $\pm 30 \mathrm{deg}$ and $\pm 40 \mathrm{deg}$, respectively.

\section{LINK-BUDGET ANALYSIS}

In this Section we analyze the link-budget for the proposed $\mathrm{mmW}$ transceiver. For simplicity, we study only the case with 1 layer as the rate will scale linearly ${ }^{1}$.

The SNR requirements are based on the model [6] and are modulation specific. When considering the EVM requirements, we adopted the following figures: QPSK $=-20 \mathrm{~dB}$, $16 \mathrm{QAM}=-20 \mathrm{~dB}, 64 \mathrm{QAM}=-26 \mathrm{~dB}$ and $256 \mathrm{QAM}=-34 \mathrm{~dB}$. The transmitted power used in link analyses is calculated from equivalent isotrophic radiated power (EIRP), which is taking into account the number of active PA elements, estimated front-end loss $(\approx 5 \mathrm{~dB})$, the gain of antenna sub-element fed from single PA, the power at PA output and the gain of antenna sub-element fed from single PA. In this implementation single PA feds 4 antenna elements in $2 \times 2$ configuration. Finally, we also include 0.85 coding.

Figure 7 shows the tradeoff between range and achievable data rate for different modulations as well as with two different level of non ideality, namely, EVM losses and EVM-TX FE losses. It can be noticed that by introducing more realistic models the range drastically reduces. Finally, if we focus on the requirements for a mobile backhaul system as described in [4], i.e. $200 \mathrm{~m}$ coverage and $2.5 \mathrm{Gbps}$, and notice that $100 \mathrm{MHz}$ with 64QAM will be sufficient to achieve this target.

\footnotetext{
${ }^{1}$ Clearly, this is true under the performance of the hybrid beamforming is close to that one achieved with a full-digital solution.
} 


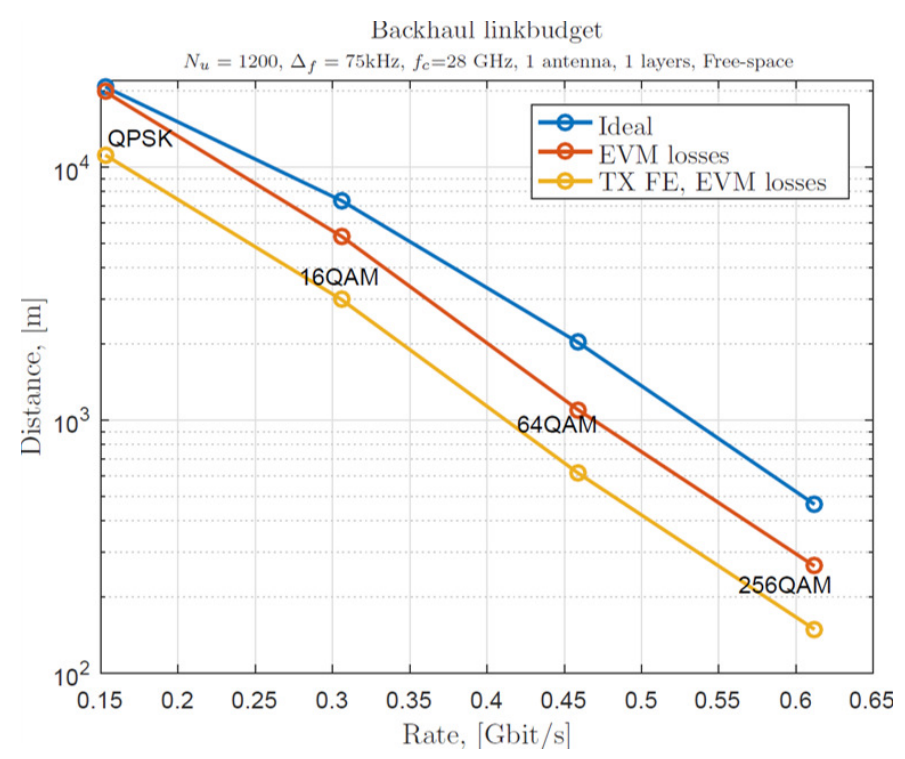

Figure 7. Link-budget for a single layer.

\section{CONCLusions}

Mobile backhaul applications require high output power and very linear devices to achieve long range and good SNR for high-order modulations. Requirements are similar to conventional base stations or fixed backhaul applications. On the other hand, mobility combined with limited total dynamic range of the $\mathrm{mmW}$ transceiver mandate very careful design of performance partitioning in the signal path as well as gain control functionality that is combined with beam steering. This paper shows key tradeoffs and solutions of those functionalities and describes transceiver architecture that can meet the requirements extending total dynamic range, i.e. usable operation distances, of a mobile backhaul link.

\section{ACKNOWLEDGMENTS}

The research leading to these results has received funding from the European Union H2020 5GPPP under grant $\mathrm{n}$. 723247 and supported by the Institute for Information \& communications Technology Promotion (IITP) grant funded by the Korea government (MSIP) (No.B0115-16-0001, 5GCHAMPION).

\section{REFERENCES}

[1] F. Boccardi, R. W. Heath, A. Lozano, T. L. Marzetta, and P. Popovski, "Five disruptive technology directions for 5G," IEEE Communications Magazine, vol. 52, no. 2, pp. 74-80, Feb. 2014.

[2] M. K. Samimi and T. S. Rappaport, "3-D Millimeter-Wave Statistical Channel Model for 5G Wireless System Design," IEEE Trans. on Microwave Theory and Techniques, vol. 64, no. 7, pp. 2207-2225, Jul. 2016.

[3] World Radiocommunication Conference (WRC-15). International Telecommunications Union (ITU), "Studies on frequency-related matters for International Mobile Telecommunications identification including possible additional allocations to the mobile services on a primary basis in portion(s) of the frequency range between 24.25 and $86 \mathrm{GHz}$ for the future development of International Mobile Telecommunications for 2020 and beyond," Tech. Rep., 2015.

[4] M. Mueck, et al, "5G CHAMPION - Rolling out 5G in 2018," in IEEE Globecom Workshops, Dec. 2016, pp. 1-6.
[5] X. Ge, et al, "5G wireless backhaul networks: challenges and research advances," IEEE Network, vol. 28, no. 6, pp. 6-11, Nov. 2014.

[6] T. Tuovinen, et al, "RF system requirement analysis and simulation methods towards $5 \mathrm{G}$ radios using massive MIMO," in $46^{\text {th }}$ European Microwave Conf. (EuMC), Oct. 2016, pp. 142-45.

[7] C. W. Kuo, et al, "An 18 to $33 \mathrm{GHz}$ Fully-Integrated Darlington Power Amplifier With Guanella-TypeTransmission-Line Transformers in 0.18 $\mu \mathrm{m}$ CMOS Technology," IEEE Microwave and Wireless Components Letters, vol. 23, no. 12, pp. 668-670, Dec. 2013.

[8] B. Park, et al, "Highly Linear mm-Wave CMOS Power Amplifier," IEEE Trans. on Microwave Theory and Techniques, vol. 64, no. 12, pp. 45354544, Dec. 2016.

[9] S. Shakib, et al, "A Highly Efficient and Linear Power Amplifier for 28-GHz 5G Phased Array Radios in 28-nm CMOS," IEEE Journal of Solid-State Circuits, vol. 51, no. 12, pp. 3020-3036, Dec. 2016.

[10] J. W. Lee and S. M. Heo, "A 27 GHz, 14 dBm CMOS Power Amplifier Using $0.18 \mu \mathrm{m}$ Common-Source MOSFETs," IEEE Microwave and Wireless Components Letters, vol. 18, no. 11, pp. 755-757, Nov. 2008

[11] R. Bhat, et al, "Large-scale power-combining and linearization in wattclass mmWave CMOS power amplifiers," in IEEE Radio Frequency Integrated Circuits Symp. (RFIC), Jun. 2013, pp. 283-286.

[12] J. H. Chen, et al, "A fully-integrated Ka-band stacked power amplifier in 45nm CMOS SOI technology," in IEEE $13^{\text {th }}$ Topical Meeting on Silicon Monolithic Integrated Circuits in RF Systems, Jan. 2013, pp. 75-77.

[13] C. F. Campbell, et al, "Design and Performance of a High Efficiency Ka-Band Power Amplifier MMIC," in IEEE Compound Semiconductor Integrated Circuit Symp. (CSICS), Oct. 2010, pp. 1-4.

[14] F. Y. Colomb and A. Platzker, " 2 and 4 watt Ka-band GaAs PHEMT power amplifier MMICs," in IEEE MTT-S Int. Microwave Symp. Digest, 2003, vol. 2, Jun. 2003, pp. 843-846.

[15] K. S. Boutros, et al, "5W GaN MMIC for Millimeter-Wave Applications," in IEEE Compound Semiconductor Integrated Circuit Symp., Nov. 2006, pp. 93-95.

[16] C. F. Campbell, et al, "High efficiency Ka-band power amplifier MMICs fabricated with a $0.15 \mu \mathrm{m} \mathrm{GaN}$ on SiC HEMT process," in IEEE/MTT-S Int. Microwave Symp. Digest, Jun. 2012, pp. 1-3.

[17] C. F. Campbell, et al, "High efficiency Ka-band Gallium Nitride power amplifier MMICs," in IEEE Int. Conf. on Microwaves, Communications, Antennas and Electronic Systems (COMCAS), Oct. 2013, pp. 1-5.

[18] S. Din, et al, "High power and high efficiency Ka band power amplifier," in IEEE MTT-S International Microwave Symp., May 2015, pp. 1-4.

[19] M. Micovic, et al, "GaN MMIC technology for microwave and millimeter-wave applications," in IEEE Compound Semiconductor Integrated Circuit Symp., Oct. 2005, pp. 173-176.

[20] P. C. Huang, et al, "A 17-35 GHz Broadband, High Efficiency PHEMT Power Amplifier Using Synthesized Transformer Matching Technique," IEEE Trans. on Microwave Theory and Techniques, vol. 60, no. 1, pp. 112-119, Jan. 2012.

[21] K. Fujii, "Low cost Ka-band 7W GaAs PHEMT based HPA with GaN PHEMT equivalent performance," in IEEE Radio Frequency Integrated Circuits Symp. (RFIC), May 2015, pp. 207-210.

[22] K. Kim and C. Nguyen, "A 16.5-28 GHz 0.18 $\mu \mathrm{m}$ BiCMOS Power Amplifier With Flat $19.4 \pm 1.2 \mathrm{dBm}$ Output Power," IEEE Microwave and Wireless Components Letters, vol. 24, no. 2, pp. 108-110, Feb. 2014.

[23] T. B. Kumar, et al, "A low power programmable gain high PAE K-/Kaband stacked amplifier in $0.18 \mu \mathrm{m}$ SiGe BiCMOS technology," in IEEE MTT-S Int. Microwave Symp., May 2015, pp. 1-4.

[24] S. Y. Mortazavi and K. J. Koh, "14.4 A Class F-1/F 24-to-31GHz power amplifier with 40.7SiGe BiCMOS," in IEEE Int. Solid-State Circuits Conf. Digest of Technical Papers (ISSCC), Feb. 2014, pp. 254-255.

[25] - "A $38 \mathrm{GHz}$ inverse class-F power amplifier with $38.5 \mathrm{~dB}$ gain, and $50 \mathrm{~mW}$ Psat in 0.13- $\mu \mathrm{m}$ SiGe BiCMOS," in IEEE Radio Frequency Integrated Circuits Symp. (RFIC), May 2015, pp. 211-214.

[26] P. J. Riemer, et al, "Ka-Band SiGe HBT Power Amplifier for SingleChip T/R Module Applications," in IEEE/MTT-S Int. Microwave Symp., Jun. 2007, pp. 1071-1074.

[27] A. Sarkar and B. Floyd, "A 28-GHz class-J Power Amplifier with 18$\mathrm{dBm}$ output power and $3514^{\text {th }}$ Topical Meeting on Silicon Monolithic Integrated Circuits in Rf Systems, Jan. 2014, pp. 71-73.

[28] N. Kalantari and J. F. Buckwalter, "A Nested-Reactance Feedback Power Amplifier for Q -Band Applications," IEEE Trans. on Microwave Theory and Techniques, vol. 60, no. 6, pp. 1667-1675, Jun. 2012. 